\title{
A discrete-time ruin model with dependence between interclaim arrivals and claim sizes
}

Zhenhua Bao ${ }^{1 *}$ and Ye Liur,2

\section{"Correspondence:} zhhbao@126.com

'School of Mathematics, Liaoning Normal University, Dalian, 116029, China

Full list of author information is available at the end of the article

\section{Springer}

\begin{abstract}
We construct a discrete-time ruin model with general premium rate and dependent setting, where the time between two occurrences depends on the previous claim size. The generating function and defective renewal equation satisfied by the Gerber-Shiu expected discounted penalty function are derived by using the roots of a generalized Lundberg's equation. Explicit expressions for the Gerber-Shiu function are obtained with discrete $K_{m}$-family claim sizes and geometric thresholds. Numerical illustration is then examined.
\end{abstract}

MSC: 62P05; $91 \mathrm{~B} 30$

Keywords: dependence; Gerber-Shiu discounted penalty function; defective renewal equation; Lundberg's equation

\section{Introduction}

In ruin theory, the compound binomial model and the risk model based on a discretetime renewal process have been extensively analyzed by [1,2], among many others. Note that, for these mentioned risk models, it is explicitly assumed that claim sizes and claim intervals are independent, which can be restrictive in practical context. Cossette et al. [3] propose a compound Markov binomial model based on the Markov Bernoulli process that introduces dependence between claim occurrences. Woo [4] analyzes a generalized Gerber-Shiu function in a discrete-time renewal risk model with an arbitrary dependence structure. Liu and Bao [5] consider a particular dependence structure among the interclaim time and the subsequent claim size and derive defective renewal equation satisfied by the Gerber-Shiu expected discounted penalty function. We mention that the dependence among claim sizes and interclaim arrivals through bivariate geometric distributions and copula functions have been investigated by Marceau [6], where explicit expressions for the Gerber-Shiu expected penalty function are derived.

As mentioned by Landriault [7], unlike the classical compound Poisson model, in which a unite premium can be assumed without loss of generality, it is clear that such reasoning does not hold for compound binomial model. In that paper, the author studies the evaluation of the generalized expected penalty function in the compound binomial risk model in which the premium rate received per period is $c\left(c \in \mathbb{N}^{+}\right)$. See also Liu and Bao [5] for a discrete-time risk model with general premium rate and time-dependent claim sizes.

(c) 2016 Bao and Liu. This article is distributed under the terms of the Creative Commons Attribution 4.0 International License (http://creativecommons.org/licenses/by/4.0/), which permits unrestricted use, distribution, and reproduction in any medium, provided you give appropriate credit to the original author(s) and the source, provide a link to the Creative Commons license, and indicate if changes were made. 
Inspired by the works [8] and [9] in a continuous-time risk process with dependence, we consider a fully discrete risk model, in which the distribution of the time until the next claim depends on the amount of the previous claim. Moreover, we further assume that the premium rate received per period is an arbitrary positive integer $c$. The surplus process of an insurance company is described as

$$
U(n)=u+c n-\sum_{i=1}^{N(n)} X_{i}
$$

where $U(0)=u \in \mathbb{N}$ is the initial surplus, $c \in \mathbb{N}^{+}$is the level premium rate, and $\{N(n), n \in$ $\mathbb{N}\}$ is the claim-counting process. The claim amounts $\left\{X_{i}, i=1,2, \ldots\right\}$ are a sequence of independent and identically distributed (i.i.d.) positive random variables with cumulative distribution function (c.d.f.) $B(\cdot)$, probability function (p.f.) $b(\cdot)$, and mean $\mu$. Assume that the random thresholds $\left\{Q_{i}, i=1,2, \ldots\right\}$ are i.i.d. with c.d.f. $H(\cdot)=1-\bar{H}(\cdot)$ and are independent of the claim sizes $\left\{X_{i}\right\}$. The distribution of the waiting time until the next claim depends on the size of the previous claim by comparing it to $\left\{Q_{i}\right\}$ as follows: If the size of the $X_{j}$ is greater than or equal to $Q_{j}$, then the time until the next claim follows geometric distribution with p.f. $\left(1-p_{1}\right) p_{1}^{n-1}, n \in \mathbb{N}^{+}$; if $X_{j}$ is less than $Q_{j}$, then the time until the next claim follows another geometric distribution with p.f. $\left(1-p_{2}\right) p_{2}^{n-1}, n \in \mathbb{N}^{+}$, where we assume that $p_{1} \neq p_{2}$ and $0<p_{i}<1, i=1,2$. Further, to guarantee that $U(n)$ has a positive drift, we assume that

$$
\frac{P(X \geq Q)}{1-p_{1}}+\frac{P(X<Q)}{1-p_{2}}>\frac{\mu}{c}
$$

which is the positive safety loading condition. Let $m_{i}(u)$ denote the Gerber-Shiu expected discounted penalty function with initial capital $u$ given that the first claim occurs according to the geometric distribution with parameter $p_{i}$, where we classify the insured as Class $i$ for convenience. More precisely, we define

$$
m_{i}(u)=E\left\{v^{T_{i}} \omega\left(U\left(T_{i}-1\right),\left|U\left(T_{i}\right)\right|\right) I\left(T_{i}<\infty\right) \mid u(0)=u\right\}, \quad u \in \mathbb{N}, i=1,2,
$$

where $0<v \leq 1$ is the discount factor, $\omega: \mathbb{N} \times \mathbb{N}^{+} \rightarrow \mathbb{N}$ is a penalty function, $I(A)$ is the indicator function of an event $A$, and $T_{i}, i=1,2$, is the time to ruin for Class $i$.

The rest of the paper is structured as follows: In Section 2, we analyze the roots of the generalized Lundberg's equation. The defective renewal equations for the Gerber-Shiu expected discounted penalty function are derived in Section 3. In Section 4, we obtain the explicit expressions for the Gerber-Shiu function when the claim sizes have discrete $K_{m}$ distributions and the random thresholds follow geometric distributions. A numerical example is also provided.

\section{Generalized Lundberg's equation}

In this section, we aim to analyze the roots of generalized Lundberg's equation, which will play an important role in deriving the renewal equation for the Gerber-Shiu function $m_{i}(u)$ defined by (1.3). Throughout the rest of the entire paper, we use the hat '^' to designate the generating function of the corresponding quantity. For simplicity, we introduce the 
following notation:

$$
\begin{aligned}
& \xi(j)=H(j) b(j), \quad j \in \mathbb{N}^{+} ; \\
& \chi(j)=\bar{H}(j) b(j)=b(j)-\xi(j), \quad j \in \mathbb{N}^{+} .
\end{aligned}
$$

By (2.1) and (2.2) we can rewrite (1.2) as

$$
\frac{\hat{\xi}(1)}{1-p_{1}}+\frac{\hat{\chi}(1)}{1-p_{2}}>\frac{\mu}{c}
$$

Denote

$$
\gamma(u)=\sum_{k=u+1}^{\infty} \omega(u, k-u) b(k), \quad u \in \mathbb{N}
$$

Then conditioning on the time and the amount of the first claim for Class 1 leads to

$$
\begin{aligned}
m_{1}(u) & =\sum_{n=1}^{\infty} v^{n}\left(1-p_{1}\right) p_{1}^{n-1}\left\{\sum_{j=1}^{u+c n}\left[m_{1}(u+c n-j) \xi(j)+m_{2}(u+c n-j) \chi(j)\right]+\gamma(u+c n)\right\} \\
& =\frac{1-p_{1}}{p_{1}} \sum_{n=1}^{\infty}\left(v p_{1}\right)^{n}\left\{\sum_{j=1}^{u+c n}\left[m_{1}(u+c n-j) \xi(j)+m_{2}(u+c n-j) \chi(j)\right]+\gamma(u+c n)\right\} .
\end{aligned}
$$

Replacing $u$ with $u-c$ in (2.5) and then rearranging the resulting equation yield

$$
m_{1}(u-c)=v\left(1-p_{1}\right) \sum_{n=0}^{\infty}\left(v p_{1}\right)^{n}\left\{\sum_{j=1}^{u+c n}\left[m_{1}(u+c n-j) \xi(j)+m_{2}(u+c n-j) \chi(j)\right]+\gamma(u+c n)\right\} .
$$

Combining (2.5) and (2.6), we get

$$
m_{1}(u-c)=v p_{1} m_{1}(u)+v\left(1-p_{1}\right)\left\{\sum_{j=1}^{u}\left[m_{1}(u-j) \xi(j)+m_{2}(u-j) \chi(j)\right]+\gamma(u)\right\} .
$$

Multiplying both sides of (2.7) by $z^{u}$ and summing over $u$ from $c$ to $\infty$ produce

$$
\begin{aligned}
z^{c} \hat{m}_{1}(z)= & v\left(1-p_{1}\right) z^{c} T_{z} \gamma(c)+v p_{1}\left[\hat{m}_{1}(z)-\sum_{u=0}^{c-1} z^{u} m_{1}(u)\right] \\
& +v\left(1-p_{1}\right)\left[\hat{m}_{1}(z) \hat{\xi}(z)-\sum_{u=0}^{c-1} z^{u} m_{1} * \xi(u)+\hat{m}_{2}(z) \hat{\chi}(z)-\sum_{u=0}^{c-1} z^{u} m_{2} * \chi(u)\right],
\end{aligned}
$$


where $*$ is the convolution factor, and $T_{z}$ is the discrete version of the Dickson-Hipp operator defined by

$$
T_{z} \gamma(c)=\sum_{u=c}^{\infty} z^{u-c} \gamma(u)=\sum_{u=0}^{\infty} z^{u} \gamma(u+c)
$$

See Li [2] for further details about the elegant properties of the discrete operator. After simple rearrangement, $(2.8)$ can be rewritten as

$$
\begin{aligned}
& {\left[z^{c}-v p_{1}-v\left(1-p_{1}\right) \hat{\xi}(z)\right] \hat{m}_{1}(z)-v\left(1-p_{1}\right) \hat{\chi}(z) \hat{m}_{2}(z)} \\
& \quad=v\left(1-p_{1}\right) z^{c} T_{z} \gamma(c)-v p_{1} \sum_{u=0}^{c-1} z^{u} m_{1}(u)-v\left(1-p_{1}\right) \sum_{u=1}^{c-1} z^{u}\left[m_{1} * \xi(u)+m_{2} *(u)\right] \\
& \quad=v\left(1-p_{1}\right) z^{c} T_{z} \gamma(c)-v \sum_{j=1}^{2} \sum_{u=0}^{c-1} m_{j}(u) f_{j, u}(z),
\end{aligned}
$$

where $f_{1, u}(z)=p_{1} z^{u}+\left(1-p_{1}\right) \sum_{y=u+1}^{c-1} z^{y} \xi(y-u)$ and $f_{2, u}(z)=\left(1-p_{1}\right) \sum_{y=u+1}^{c-1} z^{y} \chi(y-u)$ are polynomials of degree $c-1$ in $z$.

For Class 2, along very similar lines as discussed before, we obtain

$$
\begin{gathered}
{\left[z^{c}-v p_{2}-v\left(1-p_{2}\right) \hat{\chi}(z)\right] \hat{m}_{2}(z)-v\left(1-p_{2}\right) \hat{\xi}(z) \hat{m}_{1}(z)} \\
=v\left(1-p_{2}\right) z^{c} T_{z} \gamma(c)-v \sum_{j=1}^{2} \sum_{u=0}^{c-1} m_{j}(u) g_{j, u}(z),
\end{gathered}
$$

where $g_{1, u}(z)=\left(1-p_{2}\right) \sum_{y=u+1}^{c-1} z^{y} \xi(y-u)$ and $g_{2, u}(z)=p_{2} z^{u}+\left(1-p_{2}\right) \sum_{y=u+1}^{c-1} z^{y} \chi(y-u)$ are also polynomials of degree $c-1$ in $z$. Now we rewrite (2.9) and (2.10) in matrix form as

$$
(\mathbf{A}(z)-\mathbf{B}(z)) \hat{\mathbf{m}}(z)=v\left[z^{c} T_{z} \gamma(c) \mathbf{P}-\sum_{u=0}^{c-1} \mathbf{F}_{u}(z) \mathbf{m}(u)\right],
$$

where the matrices are defined respectively as $\mathbf{A}(z)=\operatorname{diag}\left(z^{c}-v p_{1}, z^{c}-v p_{2}\right), \mathbf{B}(z)=$ $\nu \mathbf{P}(\hat{\xi}(z), \hat{\chi}(z)), \mathbf{P}=\left(1-p_{1}, 1-p_{2}\right)^{T}, \mathbf{m}(u)=\left(m_{1}(u), m_{2}(u)\right)^{T}, \hat{\mathbf{m}}(z)=\left(\hat{m}_{1}(z), \hat{m}_{2}(z)\right)^{T}$, and

$$
\mathbf{F}_{u}(z)=\left(\begin{array}{ll}
f_{1, u}(z) & f_{2, u}(z) \\
g_{1, u}(z) & g_{2, u}(z)
\end{array}\right) .
$$

To ultimately invert $\hat{\mathbf{m}}(z)$, we need an expression for $\mathbf{m}(u)$ for $u=0,1, \ldots, c-1$, which depends on the roots of generalized Lundberg's equation $\operatorname{det}(\mathbf{A}(z)-\mathbf{B}(z))=0$. It is not hard to calculate that Lundberg's equation is equivalent to

$$
\hat{h}_{1, v}(z)-\hat{h}_{2, v}(z)=0 \text {, }
$$

where

$$
\hat{h}_{1, v}(z)=\left(z^{c}-v p_{1}\right)\left(z^{c}-v p_{2}\right),
$$




$$
\hat{h}_{2, v}(z)=v\left(1-p_{2}\right)\left(z^{c}-v p_{1}\right) \hat{\chi}(z)+v\left(1-p_{1}\right)\left(z^{c}-v p_{2}\right) \hat{\xi}(z) .
$$

In the following lemma, we first apply Rouché's theorem on a given contour to identify the number of roots of equation $(2.12)$ for $v \in(0,1)$.

Lemma 1 For $v \in(0,1)$, there are $2 c$ solutions of generalized Lundberg's equation (2.12), say $z_{i}=z_{i}(v)$ for $i=1,2, \ldots, 2 c$, inside the unit circle $\mathcal{C}=\{z:|z|=1\}$.

Proof For $v \in(0,1)$, it is easy to see that

$$
\begin{aligned}
\left|\hat{h}_{2}(z)\right| & \leq\left|v\left(1-p_{2}\right)\left(z^{c}-v p_{1}\right) \hat{\chi}(z)\right|+\left|v\left(1-p_{1}\right)\left(z^{c}-v p_{2}\right) \hat{\xi}(z)\right| \\
& \leq v\left(1-p_{2}\right)\left|z^{c}-v p_{1}\right| \hat{\chi}(1)+v\left(1-p_{1}\right)\left|z^{c}-v p_{2}\right| \hat{\xi}(1) \\
& <\left(1-v p_{2}\right)\left|z^{c}-v p_{1}\right| \hat{\chi}(1)+\left(1-v p_{1}\right)\left|z^{c}-v p_{2}\right| \hat{\xi}(1) .
\end{aligned}
$$

Note that $\hat{\chi}(1)+\hat{\xi}(1)=1$ and

$$
\left|z^{c}-v p_{i}\right| \geq\left|z^{c}\right|-\left|v p_{i}\right|=1-v p_{i}, \quad i=1,2 .
$$

Combining (2.15) and (2.16) yields

$$
\begin{aligned}
\left|\hat{h}_{2}(z)\right| & <\left|z^{c}-v p_{2}\right|\left|z^{c}-v p_{1}\right|(\hat{\chi}(1)+\hat{\xi}(1)) \\
& =\left|\hat{h}_{1}(z)\right| .
\end{aligned}
$$

Therefore, Rouché's theorem implies that $\hat{h}_{1, v}(z)=0$ and $\hat{h}_{1, v}(z)-\hat{h}_{2, v}(z)=0$ have the same number of zeros inside the unit circle $\mathcal{C}$. Given that $\hat{h}_{1, v}(z)=0$ has $2 c$ zeros inside $\mathcal{C}$, so does $\hat{h}_{1, v}(z)-\hat{h}_{2, v}(z)=0$.

In the case of $v=1$, the conditions of Rouché's theorem are no longer satisfied. However, we can determine the roots of (2.12) by applying Theorem 1 of Klimenok [10] as follows.

Lemma 2 For $v=1$, there are $2 c-1$ roots, say $z_{1}, \ldots, z_{2 c-1}$, to equation $(2.12)$ inside $\mathcal{C}$, in addition to the trivial root $z_{2 c}=1$.

Proof By direct calculations we obtain that

$$
\begin{aligned}
& \frac{\left.\frac{\mathrm{d}}{\mathrm{d} z} \hat{h}_{1,1}(z)\right|_{z=1}-\left.\frac{\mathrm{d}}{\mathrm{d} z} \hat{h}_{2,1}(z)\right|_{z=1}}{\tilde{h}_{1,1}(1)} \\
& \quad=\frac{c\left(1-p_{1}\right)+c\left(1-p_{2}\right)-\left[c\left(1-p_{2}\right) \hat{\chi}(1)+c\left(1-p_{1}\right) \hat{\xi}(1)+\left(1-p_{1}\right)\left(1-p_{2}\right) \mu\right]}{\left(1-p_{1}\right)\left(1-p_{2}\right)} \\
& \quad=c\left[\frac{\hat{\xi}(1)}{1-p_{1}}+\frac{\hat{\chi}(1)}{1-p_{2}}\right]-\mu>0,
\end{aligned}
$$

where the last inequality is due to the positive security loading condition (2.3). On the other hand, we can easily check that $\left|\hat{h}_{2,1}(z)\right|_{|z|=1, z \neq 1}<\left|\hat{h}_{1,1}(z)\right|_{|z|=1, z \neq 1}$. By Klimenok [10] we conclude that the number of solutions to (2.12) inside $\mathcal{C}$ is equal to $2 c-1$, that is, the 
number of roots of $\hat{h}_{1,1}(z)=0$ inside $\mathcal{C}$ minus 1 . Moreover, a trivial root to generalized Lundberg's equation (2.12) is $z_{2 c}=1$.

In what follows, we only consider the case where the roots of Lundberg's equation (2.12) are distinct. For $i=1, \ldots, 2 c$, let the nonzero row vector $\left(\mathbf{h}_{i}\right)^{T}=\left(h_{i, 1}, h_{i, 2}\right)$ be the left eigenvector of $\mathbf{A}\left(z_{i}\right)-\mathbf{B}\left(z_{i}\right)$ associated with the eigenvalue 0. Applying equation (2.11) yields

$$
\left(\mathbf{h}_{i}\right)^{T}\left[z_{i}^{c} T_{z_{i}} \gamma(c) \mathbf{P}-\sum_{u=0}^{c-1} \mathbf{F}_{u}\left(z_{i}\right) \mathbf{m}(u)\right]=0, \quad i=1, \ldots, 2 c .
$$

To identify the expression of $\mathbf{m}(u)$ for $u=0,1, \ldots, c-1$, we introduce the partitioned matrices $\mathbf{H}=\operatorname{diag}\left(\left(\mathbf{h}_{1}\right)^{T},\left(\mathbf{h}_{2}\right)^{T}, \ldots,\left(\mathbf{h}_{2 c}\right)^{T}\right)$ and $\mathbf{F}=\left(\left(\mathbf{F}\left(z_{1}\right)\right)^{T},\left(\mathbf{F}\left(z_{2}\right)\right)^{T}, \ldots,\left(\mathbf{F}\left(z_{2 c}\right)\right)^{T}\right)^{T}$ with $\mathbf{F}\left(z_{i}\right)=\left(\mathbf{F}_{0}\left(z_{i}\right), \mathbf{F}_{1}\left(z_{i}\right), \ldots, \mathbf{F}_{c-1}\left(z_{i}\right)\right), i=1,2, \ldots, 2 c$. Thus, equation (2.18) can be reexpressed as

$$
\mathbf{Q}\left(\begin{array}{c}
\mathbf{m}(0) \\
\mathbf{m}(1) \\
\vdots \\
\mathbf{m}(c-1)
\end{array}\right)=\left(\begin{array}{c}
z_{1}^{c} T_{z_{1}} \gamma(c) \sum_{l=1}^{2}\left(1-p_{l}\right) h_{1, l} \\
z_{2}^{c} T_{z_{2}} \gamma(c) \sum_{l=1}^{2}\left(1-p_{l}\right) h_{2, l} \\
\vdots \\
z_{2 c}^{c} T_{z_{2}} \gamma(c) \sum_{l=1}^{2}\left(1-p_{l}\right) h_{2 c, l}
\end{array}\right)
$$

where the matrix $\mathbf{Q}=\mathbf{H F}$.

Theorem 3 For $i=1,2$, the Gerber-Shiu expected discounted penalty function $m_{i}(u)$ with the initial surplus $u \in\{0,1, \ldots, c-1\}$ can be determined as

$$
m_{i}(u)=\sum_{j=1}^{2 c} \frac{\alpha_{j, 2 u+i}(\mathbf{Q})}{\operatorname{det} \mathbf{Q}}\left(z_{j}\right)^{c} T_{z_{j}} \gamma(c) \sum_{l=1}^{2}\left(1-p_{l}\right) h_{j, l},
$$

where $\alpha_{l, i}(\mathbf{Q})$ is the cofactor associated with the element in row l and column i of matrix $\mathbf{Q}$.

\section{Defective renewal equations}

The main goal of this section is to obtain the defective renewal equation for $m_{i}(u), i=1,2$. To do so, we solve the linear system of equations (2.9) and (2.10):

$$
\hat{m}_{1}(z)=\frac{v\left[\left(1-p_{1}\right)\left(z^{c}-v p_{2}\right) \hat{\gamma}(z)+v \hat{\chi}(z) \sum_{u=0}^{c-1} z^{u} \delta_{1}(u)-l(z)\right]}{\hat{h}_{1, v}(z)-\hat{h}_{2, v}(z)},
$$

where

$$
l(z)=\left(z^{c}-v p_{2}\right) \sum_{u=0}^{c-1}\left[m_{1}(u) f_{1, u}(z)+m_{2}(u) f_{2, u}(z)+\gamma(u)\left(1-p_{1}\right) z^{u}\right]
$$

is a polynomial of degree $2 c-1$ in $z$, and $\delta_{1}(u)=p_{1}\left(1-p_{2}\right) m_{1}(u)-p_{2}\left(1-p_{1}\right) m_{2}(u)$ for $u=0,1, \ldots, c-1$.

Since $\hat{m}_{1}(z)$ is analytic, the solutions of generalized Lundberg's equation are also zeros of the numerator in (3.1), we have

$$
l\left(z_{i}\right)=\left(1-p_{1}\right)\left(z_{i}^{c}-v p_{2}\right) \hat{\gamma}\left(z_{i}\right)+v \hat{\chi}\left(z_{i}\right) \sum_{u=0}^{c-1} z_{i}^{u} \delta_{1}(u), \quad i=1,2, \ldots, 2 c .
$$


Thus, applying the Lagrange interpolating theorem for $l(z)$ leads to

$$
\begin{aligned}
l(z) & =\sum_{j=1}^{2 c} l\left(z_{j}\right) \prod_{i=1, i \neq j}^{2 c}\left(\frac{z-z_{i}}{z_{j}-z_{i}}\right) \\
& =\pi(z)\left[\left(1-p_{1}\right) \sum_{j=1}^{2 c} \frac{\left(z_{j}^{c}-v p_{2}\right) \hat{\gamma}\left(z_{j}\right)}{\left(z-z_{j}\right) \pi^{\prime}\left(z_{j}\right)}+v \sum_{j=1}^{2 c} \frac{\hat{\chi}\left(z_{j}\right) \sum_{u=0}^{c-1} z_{j}^{u} \delta_{1}(u)}{\left(z-z_{j}\right) \pi^{\prime}\left(z_{j}\right)}\right],
\end{aligned}
$$

where $\pi(z)=\prod_{i=1}^{2 c}\left(z-z_{i}\right)$ and $\pi^{\prime}\left(z_{j}\right)=\prod_{i=1, i \neq j}^{2 c}\left(z_{i}-z_{j}\right)$. From (3.2) we conclude that

$$
\begin{aligned}
(1- & \left.p_{1}\right)\left(z^{c}-v p_{2}\right) \hat{\gamma}(z)+v \hat{\chi}(z) \sum_{u=0}^{c-1} z^{u} \delta_{1}(u)-l(z) \\
= & \pi(z)\left\{\left(1-p_{1}\right) \sum_{j=1}^{2 c} \frac{\left(z^{c}-v p_{2}\right) \hat{\gamma}(z)-\left(z_{j}^{c}-v p_{2}\right) \hat{\gamma}\left(z_{j}\right)}{\left(z-z_{j}\right) \pi^{\prime}\left(z_{j}\right)}\right. \\
& \left.+v \sum_{j=1}^{2 c} \frac{\sum_{u=0}^{c-1} \delta_{1}(u)\left[z^{u} \hat{\chi}(z)-z_{j}^{u} \hat{\chi}\left(z_{j}\right)\right]}{\left(z-z_{j}\right) \pi^{\prime}\left(z_{j}\right)}\right\} \\
= & \pi(z)\left\{\left(1-p_{1}\right) \sum_{j=1}^{2 c} \frac{\left(z_{j}^{c}-v p_{2}\right)\left(\hat{\gamma}(z)-\hat{\gamma}\left(z_{j}\right)\right)+\left(z^{c}-z_{j}^{c}\right) \hat{\gamma}(z)}{\left(z-z_{j}\right) \pi^{\prime}\left(z_{j}\right)}\right. \\
& \left.+v \sum_{j=1}^{2 c} \frac{\sum_{u=0}^{c-1}\left[\delta_{1}(u) z_{j}^{u}\left(\hat{\chi}(z)-\hat{\chi}\left(z_{j}\right)\right)+\left(z^{u}-z_{j}^{u}\right) \hat{\chi}(z)\right]}{\left(z-z_{j}\right) \pi^{\prime}\left(z_{j}\right)}\right\} \\
= & \pi(z)\left\{\left(1-p_{1}\right)\left[\sum_{j=1}^{2 c} \frac{z_{j}^{c}-v p_{2}}{\pi^{\prime}\left(z_{j}\right)} \frac{\hat{\gamma}(z)-\hat{\gamma}\left(z_{j}\right)}{z-z_{j}}+\sum_{j=1}^{2 c} \frac{\hat{\gamma}(z)}{\pi^{\prime}\left(z_{j}\right)} \frac{z^{c}-z_{j}^{c}}{z-z_{j}}\right]\right. \\
& \left.+v \sum_{u=0}^{c-1} \delta_{1}(u)\left[\sum_{j=1}^{2 c} \frac{z_{j}^{u}}{\pi^{\prime}\left(z_{j}\right)} \frac{\hat{\chi}(z)-\hat{\chi}\left(z_{j}\right)}{z-z_{j}}+\sum_{j=1}^{2 c} \frac{\hat{\chi}(z)}{\pi^{\prime}\left(z_{j}\right)} \frac{z^{u}-z_{j}^{u}}{z-z_{j}}\right]\right\} \\
& \left\{\left(1-p_{1}\right) \sum_{j=1}^{2 c} \frac{z_{j}^{c}-v p_{2}}{\pi^{\prime}\left(z_{j}\right)} T_{z} T_{z_{j}} \gamma(1)+v \sum_{u=0}^{2 c} \delta_{1}(u) \sum_{j=1} \frac{z_{j}^{u}}{\pi^{\prime}\left(z_{j}\right)} T_{z} T_{z_{j}} \chi(1)\right\}
\end{aligned}
$$

where the last equality is due to the following formula in interpolation theory:

$$
\sum_{j=1}^{m} \frac{\left(z_{j}-z\right)^{k}}{\prod_{i=1, i \neq j}^{m}\left(z_{j}-z_{i}\right)}= \begin{cases}1, & k=m-1, \\ 0, & k=0,1, \ldots, m-2 .\end{cases}
$$

Observing that $\hat{h}_{1, v}(z)$ is a polynomial of degree $2 c$ in $z$, we deduce that $\hat{h}_{1, v}(z)-\pi(z)$ is a polynomial of degree $2 c-1$ in $z$. We use the Lagrange interpolating polynomial to obtain

$$
\hat{h}_{1, v}(z)-\pi(z)=\pi(z) \sum_{i=1}^{2 c} \frac{\hat{h}_{1, v}\left(z_{j}\right)}{\pi^{\prime}\left(z_{j}\right)\left(z-z_{j}\right)} .
$$


Since $z_{j}, j=1,2, \ldots, 2 c$, are roots of generalized Lundberg's equation, we know that $\hat{h}_{1, v}\left(z_{j}\right)=$ $\hat{h}_{2, v}\left(z_{j}\right)$. Then equation (3.4) is equivalent to

$$
\hat{h}_{1, v}(z)=\pi(z)\left[1+\sum_{i=1}^{2 c} \frac{\hat{h}_{2, v}\left(z_{j}\right)}{\pi^{\prime}\left(z_{j}\right)\left(z-z_{j}\right)}\right]
$$

Therefore, we can employ a similar procedure to find an alternative expression for the denominator of the right side of (3.1) as

$$
\begin{aligned}
& \hat{h}_{1, v}(z)-\hat{h}_{2, v}(z) \\
& \quad=\pi(z)\left\{1-v\left[\left(1-p_{2}\right) \sum_{j=1}^{2 c} \frac{z_{j}^{c}-v p_{1}}{\pi^{\prime}\left(z_{j}\right)} T_{z} T_{z_{j}} \chi(1)+\left(1-p_{1}\right) \sum_{j=1}^{2 c} \frac{z_{j}^{c}-v p_{2}}{\pi^{\prime}\left(z_{j}\right)} T_{z} T_{z_{j}} \xi(1)\right]\right\} .
\end{aligned}
$$

Substituting (3.3) and (3.5) into (3.1), we deduce

$$
\begin{aligned}
\hat{m}_{1}(z) & =\frac{v\left[\left(1-p_{1}\right) \sum_{j=1}^{2 c} \frac{z_{j}^{c}-v p_{2}}{\pi^{\prime}\left(z_{j}\right)} T_{z} T_{z_{j}} \gamma(1)+v \sum_{u=0}^{c-1} \delta_{1}(u) \sum_{j=1}^{2 c} \frac{z_{j}^{u}}{\pi^{\prime}\left(z_{j}\right)} T_{z} T_{z_{j}} \chi(1)\right]}{1-v\left[\left(1-p_{2}\right) \sum_{j=1}^{2 c} \frac{z_{j}^{c}-v p_{1}}{\pi^{\prime}\left(z_{j}\right)} T_{z} T_{z_{j}} \chi(1)+\left(1-p_{1}\right) \sum_{j=1}^{2 c} \frac{z_{j}^{c}-v p_{2}}{\pi^{\prime}\left(z_{j}\right)} T_{z} T_{z_{j}} \xi(1)\right]} \\
& =\frac{v \hat{\eta}_{1}(z)}{1-v \hat{\tau}(z)},
\end{aligned}
$$

where

$$
\begin{aligned}
& \eta_{1}(i)=\left(1-p_{1}\right) \sum_{j=1}^{2 c} \frac{z_{j}^{c}-v p_{2}}{\pi^{\prime}\left(z_{j}\right)} T_{z_{j}} \gamma(1+i)+v \sum_{u=0}^{c-1} \delta_{1}(u) \sum_{j=1}^{2 c} \frac{z_{j}^{u}}{\pi^{\prime}\left(z_{j}\right)} T_{z_{j}} \chi(1+i), \quad i \in \mathbb{N}, \\
& \tau(i)=\sum_{j=1}^{2 c}\left[\left(1-p_{2}\right) \frac{z_{j}^{c}-v p_{1}}{\pi^{\prime}\left(z_{j}\right)} T_{z_{j}} \chi(1+i)+\left(1-p_{1}\right) \frac{z_{j}^{c}-v p_{2}}{\pi^{\prime}\left(z_{j}\right)} T_{z_{j}} \xi(1+i)\right], \quad i \in \mathbb{N} .
\end{aligned}
$$

For the Gerber-Shiu function $m_{2}(u)$, following a similar procedure, we have

$$
\hat{m}_{2}(z)=\frac{v \hat{\eta}_{2}(z)}{1-v \hat{\tau}(z)}
$$

where

$$
\eta_{2}(i)=\left(1-p_{2}\right) \sum_{j=1}^{2 c} \frac{z_{j}^{c}-v p_{1}}{\pi^{\prime}\left(z_{j}\right)} T_{z_{j}} \gamma(1+i)+v \sum_{u=0}^{c-1} \delta_{2}(u) \sum_{j=1}^{2 c} \frac{z_{j}^{u}}{\pi^{\prime}\left(z_{j}\right)} T_{z_{j}} \xi(1+i), \quad i \in \mathbb{N}
$$

with $\delta_{2}(u)=p_{2}\left(1-p_{1}\right) m_{2}(u)-p_{1}\left(1-p_{2}\right) m_{1}(u)$ for $u=0,1, \ldots, c-1$.

Equations (3.6) and (3.9) eventually lead to the defective renewal equation for the Gerber-Shiu discounted penalty function $m_{i}(u)$, as presented in the following theorem. 
Theorem 4 The Gerber-Shiu expected discounted penalty function $m_{i}(u)$ defined in (2.3) satisfies the following defective renewal equation:

$$
m_{i}(u)=v \sum_{j=0}^{u} m_{i}(u-j) \tau(j)+v \eta_{i}(u), \quad i=1,2,
$$

where $\tau(\cdot)$ is defined by (3.8), and $\eta_{1}(\cdot)$ and $\eta_{2}(\cdot)$ are determined by (3.7) and (3.10), respectively.

Proof The renewal equation (3.11) follows by inverting (3.6) and (3.9) directly. For (3.11) to be a defective renewal equation, it remains to show that $k_{v} \doteq v \hat{\tau}(1)<1$. Let us first assume that $v \in(0,1)$. Using $(3.5)$, we have $k_{v}=1-\frac{\hat{h}_{1, v}(1)-\hat{h}_{2, v}(1)}{\pi(1)}$. We easily calculate that

$$
\begin{aligned}
\hat{h}_{2, v}(1) & =v\left(1-p_{2}\right)\left(1-v p_{1}\right) \hat{\chi}(1)+v\left(1-p_{1}\right)\left(1-v p_{2}\right) \hat{\xi}(1) \\
& <\left(1-v p_{2}\right)\left(1-v p_{1}\right) \hat{\chi}(1)+\left(1-v p_{1}\right)\left(1-v p_{2}\right) \hat{\xi}(1) \\
& =\hat{h}_{1, v}(1)
\end{aligned}
$$

and then deduce that $k_{v}<1$.

Now we turn to the case of $v=1$. Since $z_{2 c}$ is a root of generalized Lundberg's equation (2.12), we have

$$
\hat{h}_{1, v}\left(z_{2 c}\right)-\hat{h}_{2, v}\left(z_{2 c}\right)=0 \text {. }
$$

Differentiating (3.12) with respect to $z_{2 c}$ and taking the limit $v \rightarrow 1$, we eventually find

$$
z_{2 c}^{\prime}=\frac{\left(1-p_{1}\right)\left(1-p_{2}\right)+p_{1}\left(1-p_{2}\right) \hat{\xi}(1)+p_{2}\left(1-p_{1}\right) \hat{\chi}(1)}{c\left(1-p_{2}\right) \hat{\xi}(1)+c\left(1-p_{1}\right) \hat{\chi}(1)-\left(1-p_{1}\right)\left(1-p_{2}\right) \mu}>0
$$

where the inequality is derived via (2.3). Thus,

$$
\begin{aligned}
\lim _{v \rightarrow 1} k_{v} & =1-\frac{\left(1-p_{1}\right) \hat{\chi}(1)+\left(1-p_{2}\right) \hat{\xi}(1)}{\pi^{\prime}(1)} \lim _{v \rightarrow 1} \frac{1-v}{1-z_{2 c}} \\
& =1-\frac{\left(1-p_{1}\right) \hat{\chi}(1)+v\left(1-p_{2}\right) \hat{\xi}(1)}{\pi^{\prime}(1)} \frac{1}{z_{2 c}^{\prime}}<1 .
\end{aligned}
$$

\section{Applications with geometric thresholds}

The thresholds can be viewed as a criterion for classifying claims as large or small. In this section, we suppose that the random thresholds $\left\{Q_{i}, i=1,2, \ldots\right\}$ follow a geometric distribution with p.f. $\left(1-p_{3}\right) p_{3}^{n-1}, n \in \mathbb{N}^{+}, 0<p_{3}<1$. We derive an explicit expression for the Gerber-Shiu function when the claim sizes belong to the discrete $K_{m}$-family and present some special cases.

\subsection{The Gerber-Shiu function with $K_{m}$-family claim sizes}

We assume that the claim sizes $\left\{X_{i}, i=1,2, \ldots\right\}$ follow a distribution from discrete $K_{m}$ family, that is, the generating function of the density function $b(\cdot)$ satisfies the following 
form:

$$
\hat{b}(z)=\frac{z\left[\prod_{i=1}^{m}\left(1-q_{i}\right)+\sum_{j=1}^{m-1} \beta_{j}(z-1)^{j}\right]}{\prod_{i=1}^{m}\left(1-q_{i} z\right)}, \quad \mathbf{R}(z)<\min \left\{\frac{1}{q_{i}}, 1 \leq i \leq m\right\}
$$

where $0<q_{i}<1, i=1,2, \ldots, m$, and the coefficients $\beta_{1}, \beta_{2}, \ldots, \beta_{m-1}$ are such that $b(\cdot)$ is a p.f. This class of distributions includes, as particular cases, the shifted geometric, shifted or truncated negative binomial, and linear combinations of these; see Li [2] for more examples. Let $p_{m}(z)=\prod_{i=1}^{m}\left(1-q_{i} z\right)$ and $p_{m-1}^{*}(z)=\prod_{i=1}^{m}\left(1-q_{i}\right)+\sum_{j=1}^{m} \beta_{j}(z-1)^{j}$. Then (4.1) becomes $\hat{b}(z)=\frac{z p_{m-1}^{*}(z)}{p_{m}(z)}$.

Applying definition (2.2), it follows that

$$
\hat{\chi}(z)=\sum_{y=1}^{\infty}\left(z p_{3}\right)^{y} b(y)=\hat{b}\left(p_{3} z\right) .
$$

On the other hand, we can rewrite generalized Lundberg's equation (2.12) as

$$
\begin{aligned}
\hat{h}_{1, v}(z)-\hat{h}_{2, v}(z)= & \left(z^{c}-v p_{1}\right)\left(z^{c}-v p_{2}\right)-v\left(1-p_{1}\right)\left(z^{c}-v p_{2}\right) \hat{b}(z) \\
& -v\left[\left(1-p_{2}\right)\left(z^{c}-v p_{1}\right)-\left(1-p_{1}\right)\left(z^{c}-v p_{2}\right)\right] \hat{\chi}(z) \\
= & 0 .
\end{aligned}
$$

We further denote $q_{m}(z)=p_{m}\left(p_{3} z\right)$ and $q_{m-1}^{*}(z)=p_{m-1}^{*}\left(p_{3} z\right)$. Then (4.2) can be rewritten as

$$
\begin{aligned}
& \left(z^{c}-v p_{1}\right)\left(z^{c}-v p_{2}\right)-v\left(1-p_{1}\right)\left(z^{c}-v p_{2}\right) \frac{z p_{m-1}^{*}(z)}{p_{m}(z)} \\
& \quad-v\left[\left(1-p_{2}\right)\left(z^{c}-v p_{1}\right)-\left(1-p_{1}\right)\left(z^{c}-v p_{2}\right)\right] \frac{p_{3} z q_{m-1}^{*}(z)}{q_{m}(z)}=0
\end{aligned}
$$

or, equivalently,

$$
\begin{gathered}
\left(z^{c}-v p_{1}\right)\left(z^{c}-v p_{2}\right) p_{m}(z) q_{m}(z)-v\left(1-p_{1}\right)\left(z^{c}-v p_{2}\right) z p_{m-1}^{*}(z) q_{m}(z) \\
-v p_{3}\left[\left(1-p_{2}\right)\left(z^{c}-v p_{1}\right)-\left(1-p_{1}\right)\left(z^{c}-v p_{2}\right)\right] z q_{m-1}^{*}(z) p_{m}(z)=0 .
\end{gathered}
$$

It is obvious that the left side of equation (4.3) is a polynomial of degree $2 c+2 m$ in $z$ with leading coefficient $\alpha=p_{3}^{m} \prod_{i=1}^{m} q_{i}^{2}$, which implies that there are $2 c+2 m$ roots in total. According to Lemmas 1 and 2, exactly $2 c$ roots are located in the unit circle $\mathcal{C}$, denoted by $z_{1}, z_{2}, \ldots, z_{2 c}$ as before. Therefore, we conclude that the remaining $2 m$ roots must lie in the region $\{z:|z|>1\}$, denoted by $\rho_{1}, \rho_{2}, \ldots, \rho_{2 m}$. Since the left side of (4.3) is equivalent to $p_{m}(z) q_{m}(z)\left[\hat{h}_{1, v}(z)-\hat{h}_{2, v}(z)\right]$, equation (4.3) can be reexpressed as

$$
\begin{aligned}
p_{m}(z) q_{m}(z)\left[\hat{h}_{1, v}(z)-\hat{h}_{2, v}(z)\right] & =\alpha p_{m}^{o}(z) q_{m}^{o}(z)\left[\hat{h}_{1, v}(z)-\hat{h}_{2, v}(z)\right] \\
& =\alpha \prod_{i=1}^{2 c}\left(z-z_{i}\right) \prod_{l=1}^{2 m}\left(z-\rho_{l}\right),
\end{aligned}
$$


where $p_{m}^{o}(z)=\prod_{i=1}^{m}\left(z-\frac{1}{q_{i}}\right)$ and $q_{m}^{o}(z)=\prod_{i=1}^{m}\left(z-\frac{1}{p_{3} q_{i}}\right)$ are both polynomials of degree $2 m$ in $z$ with the leading coefficient 1 . Substituting (4.4) into (3.1), we have

$$
\hat{m}_{1}(z)=v \frac{p_{m}^{o}(z) q_{m}^{o}(z)}{\prod_{l=1}^{2 m}\left(z-\rho_{l}\right)} \hat{\eta}_{1}(z)=v\left[1+\frac{S(z)}{D(z)}\right] \hat{\eta}_{1}(z)
$$

where $S(z)=p_{m}^{o}(z) q_{m}^{o}(z)-\prod_{l=1}^{2 m}\left(z-\rho_{l}\right)$ is a polynomial of degree $2 m-1$ or less, and $D(z)=$ $\prod_{l=1}^{2 m}\left(z-\rho_{l}\right)$ is a polynomial of degree $m$. Further, if $\rho_{1}, \rho_{2}, \ldots, \rho_{2 m}$ are distinct, then $\hat{g}(z) \doteq$ $\frac{S(z)}{D(z)}$ can be obtained by partial fractions as

$$
\hat{g}(z)=\sum_{i=1}^{2 m} \frac{\sigma_{i}}{\rho_{i}-z}
$$

where

$$
\sigma_{i}=\frac{\prod_{j=1}^{m}\left(\frac{1}{q_{j}}-\rho_{i}\right) \prod_{j=1}^{m}\left(\frac{1}{p_{3} q_{j}}-\rho_{i}\right)}{\prod_{j=1, j \neq i}^{2 m}\left(\rho_{j}-\rho_{i}\right)}, \quad i=1,2, \ldots, 2 m .
$$

Thus, inverting the generating function of (4.6) gives

$$
g(y)=\sum_{i=1}^{2 m} \sigma_{i} \rho_{i}^{-(y+1)}, \quad y \in \mathbb{N} .
$$

Utilizing (4.7), we can invert the generating function in (4.5) to

$$
m_{1}(u)=v\left[\eta_{1}(u)+\sum_{i=0}^{u} g(u-i) \eta_{1}(i)\right] .
$$

Similarly, from (3.9) we obtain

$$
m_{2}(u)=v\left[\eta_{2}(u)+\sum_{i=0}^{u} g(u-i) \eta_{2}(i)\right] .
$$

\subsection{The generating function of the time to ruin with geometric distribution}

In this section, we consider the generating function of the time to ruin, which is one of important quantities in risk analysis. Let the penalty function $\omega\left(x_{1}, x_{2}\right)=1$ for all $x_{1} \in \mathbb{N}$ and $x_{2} \in \mathbb{N}^{+}$. Then the Gerber-Shiu function (1.3) reduces to

$$
\varphi_{i}(u)=E\left\{v^{T_{i}} I\left(T_{i}<\infty\right) \mid U(0)=u\right\}, \quad u \in \mathbb{N}, i=1,2 .
$$

We assume that the claim sizes $\left\{X_{i}, i=1,2, \ldots\right\}$ follow geometric distribution with p.f. $b(y)=(1-q) q^{y-1}, y \in \mathbb{N}^{+}$, and the generating function $\hat{b}(z)=\frac{z(1-q)}{1-z q}$. By (2.1) and (2.2) some simple algebras lead to

$$
\hat{\chi}(z)=\frac{z p_{3}(1-q)}{1-p_{3} q z}, \quad \hat{\xi}(z)=\frac{z(1-q)\left(1-p_{3}\right)}{(1-q z)\left(1-p_{3} q z\right)} .
$$


Implementing the expressions into (4.8), generalized Lundberg's equation (2.12) becomes

$$
\begin{gathered}
\left(z^{c}-v p_{1}\right)\left(z^{c}-v p_{2}\right)-v\left(1-p_{2}\right)\left(z^{c}-v p_{1}\right) \frac{z p_{3}(1-q)}{1-p_{3} q z} \\
-v\left(1-p_{1}\right)\left(z^{c}-v p_{2}\right) \frac{z(1-q)\left(1-p_{3}\right)}{(1-q z)\left(1-p_{3} q z\right)}=0,
\end{gathered}
$$

which can be further rearranged to the following equation without changing the roots:

$$
\begin{aligned}
\left(z^{c}-v p_{1}\right)\left(z^{c}-v p_{2}\right)\left(z-\frac{1}{p_{3} q}\right)\left(z-\frac{1}{q}\right)+\frac{v(1-q)\left(1-p_{2}\right)}{q}\left(z-\frac{1}{q}\right)\left(z^{c}-v p_{1}\right) \\
+\frac{v\left(1-p_{1}\right)(1-q)\left(1-p_{3}\right) z\left(z^{c}-v p_{2}\right)}{q^{2} p_{3}}=0 .
\end{aligned}
$$

As discussed in Section 4.1, there are two roots lying in the complex plane $\{z:|z|>1\}$, denoted by $\rho_{1}, \rho_{2}$. Henceforth, we assume that $\rho_{1} \neq \rho_{2}$. The other $2 c$ roots are in the unit circle $\mathcal{C}$, still denoted by $z_{i}, i=1,2, \ldots, 2 c$. Moreover, we have

$$
\left(z-\frac{1}{p_{3} q}\right)\left(z-\frac{1}{q}\right)\left[\hat{h}_{1, v}(z)-\hat{h}_{2, v}(z)\right]=\prod_{i=1}^{2 c}\left(z-z_{i}\right)\left(z-\rho_{1}\right)\left(z-\rho_{2}\right) .
$$

On the other hand, substituting (4.8) into the expression of $\hat{\eta}_{1}(z)$ finally proves that

$$
\hat{\eta}_{1}(z)=-\left[\frac{\kappa_{1}}{z-\frac{1}{q}}+\frac{\kappa_{2}}{z-\frac{1}{p_{3} q}}\right]
$$

where the constants $\kappa_{1}$ and $\kappa_{2}$ are determined respectively by

$$
\begin{aligned}
& \kappa_{1}=\left(1-p_{1}\right) \sum_{j=1}^{2 c} \frac{z_{j}^{c}-v p_{2}}{\pi^{\prime}\left(z_{j}\right)\left(q z_{j}-1\right)}, \\
& \kappa_{2}=\frac{v(1-q)}{q} \sum_{u=0}^{c-1} \delta_{1}(u) \sum_{j=1}^{2 c} \frac{z_{j}^{u}}{\pi^{\prime}\left(z_{j}\right)\left(p_{3} q z_{j}-1\right)} .
\end{aligned}
$$

Inserting (4.10) and (4.11) into (3.6), we obtain

$$
\hat{\varphi}_{1}(z)=-\frac{v\left[\kappa_{1}\left(z-\frac{1}{p_{3} q}\right)+\kappa_{2}\left(z-\frac{1}{q}\right)\right]}{\left(z-\rho_{1}\right)\left(z-\rho_{2}\right)} .
$$

Using partial fractions, we rewrite (4.12) as

$$
\hat{\varphi}_{1}(z)=\sum_{i=1}^{2} \frac{\lambda_{i}}{\rho_{i}-z},
$$

where

$$
\lambda_{i}=\frac{v\left[\kappa_{1}\left(\rho_{i}-\frac{1}{p_{3} q}\right)+\kappa_{2}\left(\rho_{i}-\frac{1}{q}\right)\right]}{\rho_{i}-\rho_{j}}, \quad i, j=1,2, i \neq j .
$$


Finally, the inversion of (4.11) yields

$$
\varphi_{1}(u)=\sum_{i=1}^{2} \lambda_{i} \rho_{i}^{-(u+1)}
$$

As for $\varphi_{2}(u)$, direct calculations yield

$$
\hat{\varphi}_{2}(z)=-\frac{v\left[\iota_{1}\left(z-\frac{1}{p_{3} q}\right)-\iota_{2} z+\iota_{3}\right]}{\left(z-\rho_{1}\right)\left(z-\rho_{2}\right)},
$$

where the constants $\iota_{i}, i=1,2,3$, are given by

$$
\begin{aligned}
& \iota_{1}=\left(1-p_{2}\right) \sum_{j=1}^{2 c} \frac{z_{j}^{c}-v p_{1}}{\pi^{\prime}\left(z_{j}\right)\left(q z_{j}-1\right)}, \\
& \iota_{2}=v(1-q)\left(1-p_{3}\right) \sum_{u=0}^{c-1} \delta_{2}(u) \sum_{j=0}^{2 c} \frac{z_{j}^{u+1}}{\pi^{\prime}\left(z_{j}\right)\left(q z_{j}-1\right)\left(p_{3} q z_{j}-1\right)}, \\
& \iota_{3}=\frac{v(1-q)\left(1-p_{3}\right)}{q^{2} p_{3}} \sum_{u=0}^{c-1} \delta_{2}(u) \sum_{j=0}^{2 c} \frac{z_{j}^{u}}{\pi^{\prime}\left(z_{j}\right)\left(q z_{j}-1\right)\left(p_{3} q z_{j}-1\right)} .
\end{aligned}
$$

Consequently, $\varphi_{2}(u)$ can be obtained by partial fractions as

$$
\varphi_{2}(u)=\sum_{i=1}^{2} \sigma_{i} \rho_{i}^{-(u+1)}
$$

where

$$
\sigma_{i}=\frac{v\left[\iota_{1}\left(\rho_{i}-\frac{1}{p_{3} q}\right)-\iota_{2} \rho_{i}+\iota_{3}\right]}{\rho_{i}-\rho_{j}}, \quad i, j=1,2, i \neq j
$$

Example In this example, we assume that the thresholds $\left\{Q_{i}\right\}$ and claim sizes $\left\{X_{i}\right\}$ both follow geometric distributions with parameters $p_{3}=0.2$ and $q=0.6$. Let $c=1, p_{1}=0.7$, $p_{2}=0.8, v=0.85$. It is easy to check that the positive loading condition is fulfilled. Generalized Lundberg's equation has four roots: $z_{1}=0.7410, z_{2}=0.6601, \rho_{1}=1.3692, \rho_{2}=8.3914$. Furthermore, we use (4.14) and (4.15) to calculate the exact values for $\varphi_{i}(u)$ :

$$
\begin{aligned}
& \varphi_{1}(u)=0.3491 \times 1.3692^{-(u+1)}-0.0016 \times 8.3914^{-(u+1)}, \\
& \varphi_{2}(u)=0.5969 \times 1.3692^{-(u+1)}-0.0228 \times 8.3914^{-(u+1)} .
\end{aligned}
$$

Figure 1 describes the behavior of $\varphi_{i}(u), i=1,2$, with respect to initial surplus $u$. As expected, $\varphi_{i}(u)$ decreases as the initial surplus $u$ increases. Moreover, $\varphi_{1}(u)$ is always smaller than $\varphi_{2}(u)$.

\section{Conclusions}

We study a discrete-time ruin model with general premium rate and dependence structure, in which the distribution of the time until the next claim depends on the amount 
Figure 1 The generating function of the time to ruin: $\varphi_{1}(u)$ (dashed line) and $\varphi_{2}(u)$ (solid line).

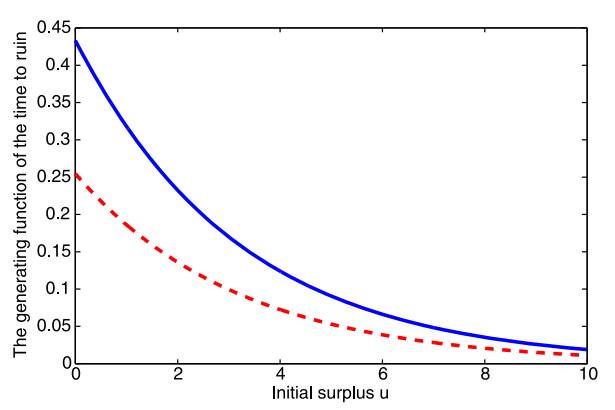

of the previous claim. Some analytic techniques are applied to study the Gerber-Shiu expected discounted penalty function. In particular, we show that the Gerber-Shiu function satisfies a defective renewal equation. Explicit expressions for the Gerber-Shiu function are obtained with discrete $K_{m}$-family claim sizes and geometric thresholds. The model in this paper can be further extended. For instance, suppose that the premium charged varies depending on the possible change in the distribution of interclaim time. Then the related ruin problems can be solved for this modified model.

\section{Competing interests}

The authors declare that there is no conflict of interests regarding the publication of this paper.

\section{Authors' contributions}

All authors have equal contributions. All authors read and approved the final manuscript.

\section{Author details}

'School of Mathematics, Liaoning Normal University, Dalian, 116029, China. ${ }^{2}$ Songjiang Jiuting No. 4 Primary School, Shanghai, 201615, China.

\section{Acknowledgements}

The authors are very grateful to the anonymous referee for his/her valuable comments and suggestions. This research was supported by the Ministry of Education of Humanities and Social Science Project (15YJC910001) and the Program for Liaoning Excellent Talents in University (LR2014031).

Received: 26 November 2015 Accepted: 9 June 2016 Published online: 11 July 2016

\section{References}

1. Cheng, S, Gerber, HU, Shiu, ESW: Discounted probabilities and ruin theory in the compound binomial model. Insur. Math. Econ. 26, 239-250 (2000)

2. Li, S: On a class of discrete time renewal risk models. Scand. Actuar. J. 2005, 241-260 (2005)

3. Cossette, H, Landriault, D, Marceau, E: Ruin probabilities in the compound Markov binomial model. Scand. Actuar. J. 2003, 301-323 (2003)

4. Woo, JK: A generalized penalty function for a class of discrete renewal processes. Scand. Actuar. J. 2012, 130-152 (2012)

5. Liu, H, Bao, Z: On a discrete-time risk model with general income and time-dependence claims. J. Comput. Appl. Math. 260, 470-481 (2014)

6. Marceau, E: On the discrete-time compound renewal risk model with dependence. Insur. Math. Econ. 44, 245-259 (2009)

7. Landriault, D: On a generalization of the expected discounted penalty function in a discrete-time insurance risk model. Appl. Stoch. Models Bus. Ind. 24, 525-539 (2008)

8. Albrecher, H, Boxma, OJ: A ruin model with dependence between claim sizes and claim intervals. Insur. Math. Econ. $35,245-254(2004)$

9. Li, Z, Sendova, KP: On a ruin model with both interclaim times and premiums depending on claim sizes. Scand. Actuar. J. 2013, 1-21 (2013)

10. Klimenok, V: On the modification of Rouché's theorem for the queuing theory problems. Queueing Syst. 38, 431-434 (2001) 